\title{
Effect of the localization on the response of a quasi-periodic electromagnetic oscillator array for vibration energy harvesting
}

\author{
Kaouthar Aouali ${ }^{1,2, *}$, Najib Kacem ${ }^{1}$, Elyes Mrabet $^{2}$, Noureddine Bouhaddi ${ }^{1}$ and Mohamed Haddar $^{2}$ \\ ${ }^{1}$ Univ. Bourgogne Franche-Comté, FEMTO-ST Institute, CNRS/UFC/ENSMM/UTBM, Department of Applied Mechanics, \\ 25000 Besançon, France \\ ${ }^{2}$ National Engineering School of Sfax, Research Laboratory of Mechanics Modeling and Production, Sfax, Tunisia
}

\begin{abstract}
Vibration energy harvesting by exploiting the multimodal approach in a quasi-periodic system is proposed. The quasi-periodic system, based on electromagnetic transduction, consists of two weakly coupled magnets mechanically guided by two elastic beams. Mistuning is achieved by varying the mechanical stiffness of one of the beams. These imperfections will lead to the vibration energy localization in regions close to the imperfections which will be exploited to maximize the harvested energy.
\end{abstract}

\section{Introduction}

The diversity of ambient sources of energy arouses researchers to make its scavenging a focus of interest. Many efforts have, recently, been made to develop techniques for vibration energy harvesting based on various energy conversion mechanisms. In the following, we are interested in a vibration energy harvester (VEH) by exploiting the multimodal approach [1], which involves operating multiple modes in a periodic system. Depending on the magnitude of the disorder and the strength of the internal coupling of the system, it is possible to localize the vibration energy in regions close to the imperfections [4]. Thus, we introduce an irregularity in the mechanical stiffness of one of the two beams. This phenomenon will be exploited to maximize the harvested energy. Numerical simulations have been performed to highlight the benefits of the localization on the VEH in terms of harvested power. Thus, the optimal mistuning can be determined in order to enhance the performances of the proposed device.

\section{System modelling}

\subsection{Design}

The proposed harvester, inspired from the work of Mahmoudi and al [2] and depicted in figure 1, is based on magnetic transduction. It is composed of two weakly coupled magnets guided by elastic beams. The magnetic poles are oriented in such a way that repulsive forces are created between each two adjacent magnets. These forces lead to "nonlinear magnetic stiffness". Wire-wound copper coils are wrapped around the moving magnets. When the device is subjected to a harmonic base excitation, each moving magnet oscillates around its equilibrium position and a current is induced in each coil.

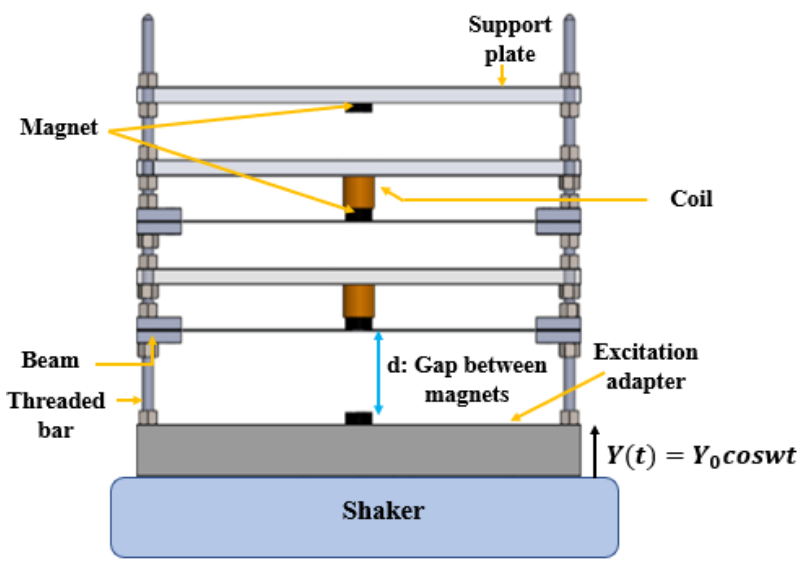

Fig. 1. Design of the proposed vibration energy harvester

The design and physical parameters are reported in Table 1.

\subsection{Problem formulation}

In this study, the fourth order partial differential equations of the continuum system are derived using the Hamilton principle. Then, a reduced-order model is generated by Galerkin method, transforming the system into a finitedegree-of-freedom system in terms of generalized coordinates.

\section{Solution procedure}

\subsection{Equation of motion}

* Corresponding author: aouali.kaouthar@gmail.com 
After normalization and generation of a reduced order model, the coupled multi-physics problem, for a quasiperiodic system of two degrees of freedom (dof), that its equivalent model shown in figure 2 , can be written in dimensionless form of modal amplitudes $A_{1}, A_{2}$ as:

$$
\left\{\begin{array}{l}
M_{e q 1} \ddot{a}_{1}+c \dot{a}_{1}+\left(k_{m e c 1}+2 k_{m a g}\right) a_{1}-k_{\text {mag }} a_{2}=-\left(M_{e q 1}+m_{1}\right) \ddot{Y} \\
\alpha M_{e q 1} \ddot{a}_{2}+c \dot{a}_{2}+\left(k_{m e c 1}+2 k_{m a g}\right) a_{2}-k_{m a g} a_{1}=-\left(\alpha M_{e q 1}+m_{1}\right) \ddot{Y}_{(1)} \\
i_{k}(t)=\frac{\delta}{\left(R_{\text {load }}+R_{\mathrm{int}}\right)} a_{k}, \quad k=1,2
\end{array}\right.
$$

where $M_{e q 1}$ is the equivalent mass of the $k^{\text {th }}$ dof, $c$ is the sum of the mechanical and the electrical damping, $k_{\text {meci }}$ is the mechanical stiffness, $k_{m g}$ is the magnetic stiffness, $m_{1}$ is the mass, $\ddot{Y}$ is the base excitation, $R_{\text {load }}$ is the load resistance and $R_{\text {int }}$ is the internal resistance and $\delta$ is the electromechanical coupling coefficient.

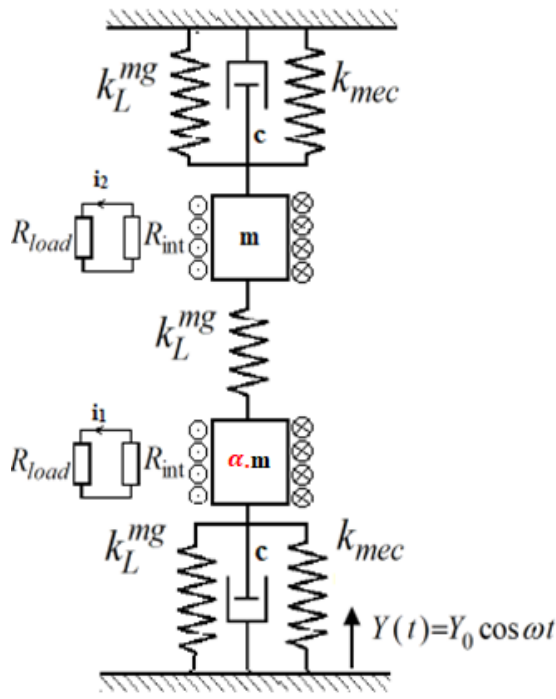

Fig. 2. Equivalent model of the proposed vibration energy harvester

The response of the system is expressed as follows:

$$
\left\{\begin{array}{cc}
\left(\begin{array}{cc}
-\Omega^{2}+j \Gamma \Omega+(1+2 \beta) & -\beta \\
-\beta & -\alpha \Omega^{2}+i \Gamma \Omega+(1+2 \beta)
\end{array}\right)\left(\begin{array}{l}
A_{1} \\
A_{2}
\end{array}\right)=\left(\begin{array}{l}
(1+\mu) f \Omega^{2} \\
(\alpha+\mu) f \Omega^{2}
\end{array}\right) \\
i_{k}(t)=\frac{\delta}{\left(R_{\text {load }}+R_{\text {int }}\right)} a_{k} & k=1,2 ; j^{2}=-1
\end{array}\right.
$$

where $\alpha$ is the mistuning, $\mu$ is the mass ratio, $\beta$ is the coupling factor, $\Omega$ is the frequency ratio, $\Gamma$ is the damping factor and $f$ is the dimensionless base excitation.

According to Mann and Sims [3], the electrical damping is defined as follows:

$$
c_{e}=\frac{\delta^{2}}{R_{\text {load }}+R_{\mathrm{int}}}
$$

Maximum dimensionless amplitudes are expressed as follows:

$$
\left\{\begin{array}{l}
A_{1}=\frac{(1+p)\left(-\alpha \Omega^{2}+i \Gamma \Omega+2 \beta+1\right)+\beta(\alpha+p)}{\left(-\Omega^{2}+i \Gamma \Omega+2 \beta+1\right) \times\left(-\alpha \Omega^{2}+i \Gamma \Omega+2 \beta+1\right)-\beta^{2}} \times Y_{0} \Omega^{2} \\
A_{2}=\frac{(\alpha+p)\left(-\alpha \Omega^{2}+i \Gamma \Omega+2 \beta+1\right)+\beta(1+p)}{\left(-\Omega^{2}+i \Gamma \Omega+2 \beta+1\right) \times\left(-\alpha \Omega^{2}+i \Gamma \Omega+2 \beta+1\right)-\beta^{2}} \times Y_{0} \Omega^{2}
\end{array}\right.
$$

\subsection{Harvested power and modal localization}

The induced current flowing in the load resistance provides an electric power. In order to highlight the effects of mistuning by mean of maximization of both the harvested power and the difference between maximum peaks amplitudes, we define, then, the harvested power and the modal localization ratio respectively as follows:

$$
\begin{aligned}
& \bar{P}=R_{\text {load }}\left[\frac{\delta}{\left(R_{\text {load }}+R_{\text {int }}\right)}\right]^{2}\left[\sum_{\alpha, \beta, R_{\text {load }}} \omega_{i}^{2}(\alpha, \beta) A_{\text {imax }}^{2}\left(\alpha, \beta, R_{\text {load }}\right)\right](5) \\
& \text { and } \quad \tau_{L}=\frac{\left|A_{1 \max }-A_{2 \max }\right|}{\max \left(A_{1 \max }, A_{2 \max }\right)}
\end{aligned}
$$

\section{Parametric study}

The variation of nondimensional amplitudes of vibrations of the two magnets with mistuning factor is depicted in figure 2 . We notice that, these amplitudes are equal when $\alpha=1$. This simulation is run with these parameters: $Y_{0}=$ 2. $10^{-2}, R_{\text {load }}=12 \Omega, d=50 \mathrm{~mm}$.

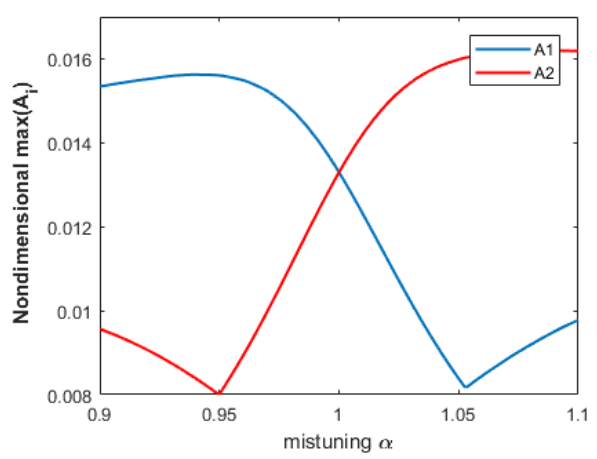

Fig. 2. Maximum dimensionless amplitudes with mistuning

In order to highlight benefits of localization, two configurations to harvest the vibration energy have been proposed. The first one aims to harvest energy from the 
vibrations of the two moving magnets. And, the second one aims to harvest energy from the vibrations of the mistuned magnet.

Results of the first and second configurations are shown in figures 3, 4, 5 and 6.

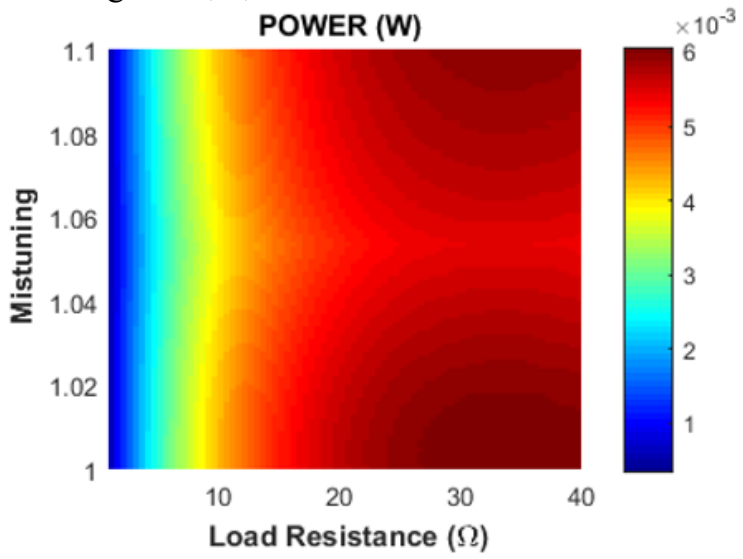

Fig. 3. Harvested power with mistuning and load resistance (configuration 1)

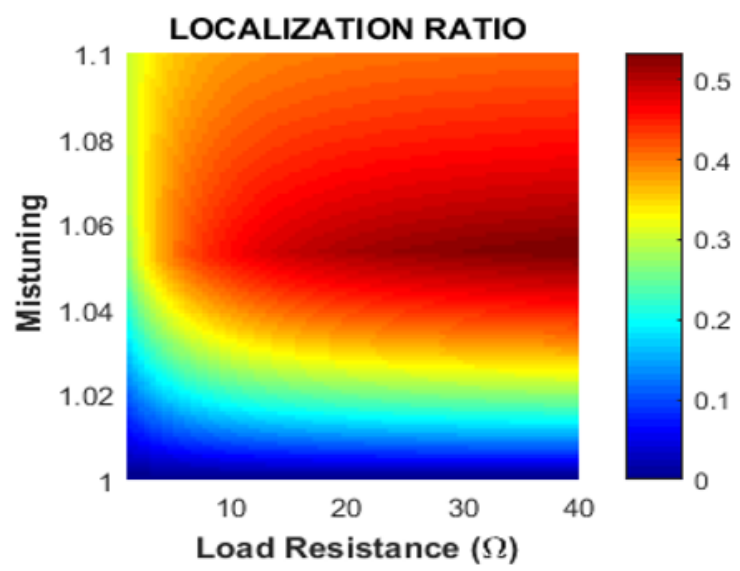

Fig. 4. Localization ratio with mistuning and load resistance (configuration 1)

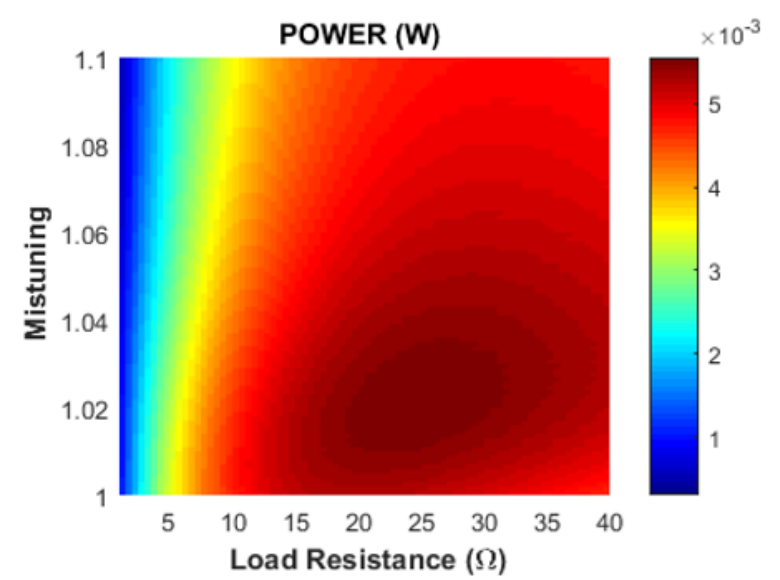

Fig. 5. Harvested power with mistuning and load resistance (configuration 2)

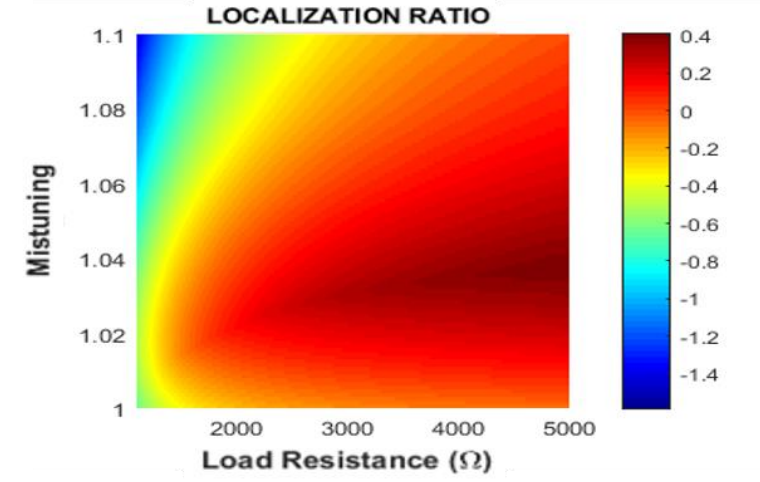

Fig. 6. Localization ratio with mistuning and load resistance (configuration 2)

For the harvested power, we notice that for the two configurations, the results are comparable. As for the modal localization, its ratio decreases slightly. The results of the second configuration compared to the first one prove the benefits of the localization phenomenon. Add to that, this configuration is more attractive in terms of technological constraints and cost. For that, it will be the configuration adopted throughout the following study.

\section{Multiobjective optimization}

Having already adopted the second configuration, we propose to conduct a multiobjective optimization aimed at maximizing simultaneously the harvested power and the modal localization ratio. For this purpose, we define the multiobjective problem as follows:

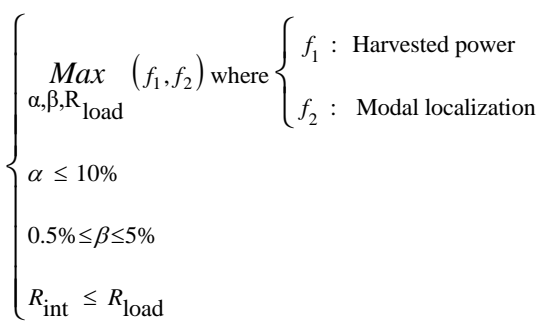

In the objective functions space, we plot the Pareto front which shows the variation of a function with the second one. As maximizing both functions is our purpose, we choose, then, values which ensure a compromise between the two objectives. The Pareto front is depicted in the figure 7.

\footnotetext{
Corresponding author: aouali.kaouthar@gmail.com
} 


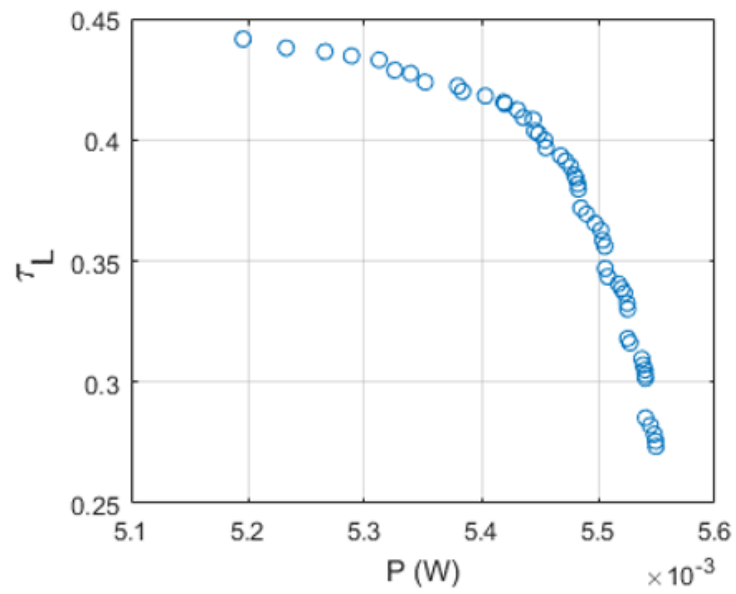

Fig. 7. The optimal solutions on the Pareto front

Then, the results of the multiobjective optimization procedure are shown in the following system:

$$
\left\{\begin{array}{l}
\underset{\alpha, \beta, R_{\text {load }}}{\operatorname{Max}}\left(\bar{P}, \tau_{L}^{b f}\right)=(5.4 m W, 41 \%) \\
\alpha^{*} \in[1.03,1.04] \\
\beta^{*} \in[1.5 \%, 2.2 \%] \\
R_{\text {load }}^{*} \in[20 \Omega, 25 \Omega]
\end{array}\right.
$$

Table 1. Design parameters

\begin{tabular}{|c|c|c|}
\hline Parameter & Designation & Value \\
\hline $\mathrm{L}(\mathrm{m})$ & Beam Length & $6010^{-3}$ \\
\hline $\mathrm{b}(\mathrm{m})$ & Beam width & $410^{-3}$ \\
\hline $\operatorname{Lc}(\mathrm{m})$ & Magnet Length & $510^{-3}$ \\
\hline $\mathrm{hs}(\mathrm{m})$ & Beam thickness & $0.610^{-3}$ \\
\hline $\mathrm{d}(\mathrm{m})$ & Gap between magnets & $5010^{-3}$ \\
\hline $\mathrm{M}(\mathrm{Kg})$ & Beam mass & $6010^{-3}$ \\
\hline$\delta(\mathrm{Vs} / \mathrm{m})$ & $\begin{array}{l}\text { Parameter of magnet } \\
\text { and coil }\end{array}$ & 1.1 \\
\hline $\mathrm{S}(\mathrm{m})$ & Magnet surface & $2610^{-4}$ \\
\hline$\rho\left(\mathrm{Kg} / m^{3}\right)$ & Steel's Density & 7800 \\
\hline $\operatorname{Rint}(\Omega)$ & Internal resistance & 4.3 \\
\hline $\mathrm{E}(\mathrm{Pa})$ & $\begin{array}{l}\text { Steel's Young } \\
\text { modulus }\end{array}$ & $21010^{9}$ \\
\hline
\end{tabular}

